\title{
Cuban medical collaborations: Contextual and clinical challenges
}

\author{
M Motala, MB ChB, MMedSci; J M van Wyk, BSc (Ed), BEd, MEd, PhD \\ Department of Clinical and Professional Practice, Nelson Mandela School of Medicine, Faculty of Health Sciences, University of KwaZulu-Natal, Durban, South Africa
}

Corresponding author: JMvan Wyk(vanwykj2@ukzn.ac.za)

\section{Context and setting}

Cuban medical internationalism ${ }^{[1]}$ is a strategy aimed at providing medical personnel to under-resourced countries. To address the severe shortage of medical doctors in rural South Africa (SA), our government forged an intergovernmental agreement to train rural and disadvantaged students at tertiary Cuban medical facilities. The collaboration students spend a year studying Spanish, and then receive an additional 4 years of medical training before returning to SA to complete their 5 th and final academic years. They graduate from a local medical school with a view to practise in rural and underserved areas of SA. This report summarises a study that explored the success of the collaboration strategy at a local medical school.

\section{Why the idea was necessary}

Despite the implementation of a learner support programme, general observations indicate that approximately two-thirds of each returning class (averaging 10 - 13 students) are unable to complete their training within the dedicated 18 months after returning to SA. Generally, the students have difficulty integrating and applying their knowledge and skills on the SA clinical platform; they struggle to translate their knowledge into English; and they have had insufficient exposure to common SA diseases.

\section{What was done?}

To strengthen and support the collaboration students, this quantitative study used questionnaires and interviews to investigate the curricular experience and teaching exposures in clinical skills of the collaboration students before their return to SA. All the students were invited and participated $(N=11)$. Institutional ethical approval was obtained.

\section{Results and impact}

Findings revealed that the Cuban curriculum emphasises primary healthcare and training in clinical settings, which focus predominantly on preventive care. The healthcare philosophy also considers the disease profiles prevalent in the Cuban setting, thus leaving students inadequately prepared for clinical practice in SA. The majority of students displayed gaps in their knowledge and an inadequate performance of clinical skills. ${ }^{[2]}$ The students found it difficult to translate the concepts learnt in Spanish into English medical terminology, and they reported inadequate knowledge of infectious diseases, specifically HIV/AIDS and tuberculosis.

Knowledge and understanding of the context of healthcare delivery, including population characteristics and disease profiles, impact on curricular decisions. Treating and managing infectious diseases that are prevalent in SA require consideration and communication between participating institutions to ensure adequate exposure and training for practice in SA conditions.

SA institutions are socially accountable to train doctors appropriately to respond to the needs of local communities. It is therefore important to support the academic needs and requirements of the collaboration students to ensure their successful education and training. This study identified areas where students lacked knowledge and skills, which informed the design and content of the support programme offered at our institution.

\section{Conclusion}

The study has highlighted the need to compare educational exposures and clinical experiences of students at our institution with those of the collaboration students to inform the details required in the support programme. Cuban medical internationalism may benefit countries with a similar language, patient profile and health contexts. This strategy, however, has limitations in SA, as it requires schools to allocate additional human and financial resources to support the collaboration students' adaptation to the local context.

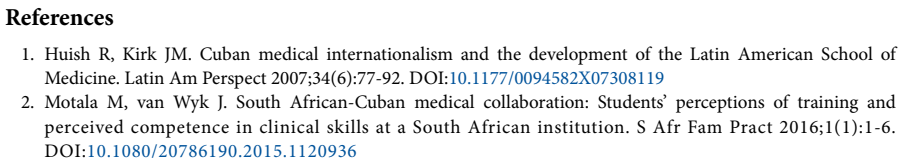

1. Huish R, Kirk JM. Cuban medical internationalism and the development of the Latin American School of Medicine. Latin Am Perspect 2007;34(6):77-92. DOI:10.1177/0094582X07308119

2. Motala M, van Wyk J. South African-Cuban medical collaboration: Students' perceptions of training and perceived competence in clinical skills at a South African institution. S Afr Fam Pract 2016;1(1):1-6. DOI:10.1080/20786190.2015.1120936

Afr J Health Professions Educ 2016;8(2):129. DOI:10.7196/AJHPE.2016.v8i2.641 